\title{
Technology Development and Commercialization Branch
}

National Cancer Institute

\section{Source}

National Cancer Institute. Technology Development and Commercialization Branch. NCI

Thesaurus. Code C20041.

Oversees and coordinates technology transfer and partnering with the federal government to develop leading-edge technologies into products that improve public health. 